\title{
Evaluation and Characterization of Pseudo-stationary Phases in Micellar Electrokinetic Chromatography
}

\author{
P. G. Muijselaar ${ }^{1} * /$ H. A. Claessens / C. A. Cramers
}

Laboratory of Instrumental Analysis, Eindhoven University of Technology, P.O. Box 513, 5600 MB Eindhoven, The Netherlands

${ }^{1}$ Present address: Faculty of Science, Himeji Institute of Technology, Kamigori, Hyogo 678-12, Japan

\section{Key Words}

MEKC

Pseudo-stationary phases

The separation mechanism of neutral species in Micellar Electrokinetic Chromatography (MEKC) is based on differences in the partitioning between the aqueous mobile phase and the pseudo-stationary micellar phase $[1,2]$. An important feature of MEKC is its flexibility. The composition of the electrolyte system can easily be changed in order to control migration behaviour and optimize selectivity. In this respect the pseudo-stationary phase plays a key role, since its chemical nature has a major influence on the separation process. Various surfactant systems can be used as well as mixed micelles, possessing different solubilization characteristics.

Despite the ease of varying the experimental conditions, proper selection of a suitable surfactant system in MEKC is still a difficult task. In gas chromatography stationary phases can be chosen on the basis of specific selective chemical interactions using the Rohrschneider-McReynolds scale [3], whereas in liquid chromatography mobile phases can be selected based on Snyder's selectivity triangle [4]. At present, however, the influence of the chemical nature of the pseudo-stationary phase and the structural properties of solutes on solute-micelle interactions in MEKC is still not well understood. Terabe and Okada studied the solubilization characteristics of several surfactant systems by the determination of thermodynamic quantities [5]. Recently, Yang and Khaledi applied Linear Solvation Energy Relationship (LSER) modelling for the characterization of solute-micelle interactions [6, 7]. LSER studies are also based on a thermodynamic approach and provide quantitative information about different solute-micelle interaction phenomena. They can be used to elucidate which mechanisms play a dominant role in

Presented at the $21^{\text {st }}$ ISC held in Stuttgart, Germany, $15^{\text {th }}-20^{\text {th }}$ September, 1996
MEKC selectivity. In this work the retention index concept is applied for the quantitative characterization of pseudo-stationary phases in MEKC.

Previously we demonstrated that the retention index, $I$, is a good migration parameter to compare different surfactant systems since $I$ is independent of the concentration, molar volume and critical micelle concentration of the applied surfactant [8]. For a given sample compound in a specific surfactant system, $S F$, a $\Delta I_{S F}$ value can be calculated according to:

$$
\Delta \mathrm{I}_{S F}=I_{S F}-I_{S D S}
$$

where $I_{S F}$ is the retention index obtained with that specific surfactant system and $I_{S D S}$ is the retention index obtained with sodium dodecylsulphate (SDS). Here SDS is chosen as reference system because this is the most widely used surfactant system in MEKC. These $\Delta I_{S F}$ values yield information about the influence of different solute functionalities and micelle characteristics on solute-micelle interactions.

Analogous to the Rohrschneider-McReynolds scale in GC [3], $\Delta I_{S F}$ values can be applied for the classification of pseudo-stationary phases in MEKC. From the LSER models, reported by Yang and Khaledi $[6,7]$, and from own measurements it can be concluded that for several surfactant systems differences in selectivity are primarily due to their hydrogen bonding abilities. Hence for these surfactant systems differences in retention indices of a solute can be expressed in terms of two parameters, representing the hydrogen bond acceptor (HBA) and hydrogen bond donor (HBD) strength of both the solute and the micellar phase, according to:

$$
\Delta I_{S F}=\beta x+\alpha y
$$

where $\beta$ and $\alpha$ are physical quantities which represent the HBA and HBD strength of the solute, and $x$ and $y$ are system constants which represent the HBD and HBA strength of the micellar pseudo-stationary phase, respectively. In order to evaluate these system constants 
Table II. System constants $x$ and $y$ for five anionic and two cationic micellar systems.

\begin{tabular}{|lrc|}
\hline Surfactant system & \multicolumn{1}{c|}{$x$} & $y$ \\
\hline 50 mM sodium dodecylsulphate & 0 & 0 \\
$50 \mathrm{mM}$ TRIS dodecylsulphate & 5 & -3 \\
$50 \mathrm{mM}$ sodium dodecylsulphate & -39 & 36 \\
$50 \mathrm{mM}$ sodium dodecylsulphate $+2 \mathrm{mM}$ Brij 35 & -87 & 48 \\
$50 \mathrm{mM}$ sodium dodecylsulphate $+5 \mathrm{mM}$ Brij 35 & -154 & 105 \\
$50 \mathrm{mM}$ sodium dodecylsulphate $+10 \mathrm{mM}$ Brij 35 & -180 & 135 \\
$50 \mathrm{mM}$ cetyltrimethylammonium bromide & -185 & 300 \\
$50 \mathrm{mM}$ dodecyltrimethylammonium bromide & -185 & 293 \\
\hline
\end{tabular}

Table I. Solvatochromic parameters $\beta$ and $\alpha$ for acetophenone and phenol in Eq. (2). [9].

\begin{tabular}{|lcc|}
\hline Standard & $\beta$ & $\alpha$ \\
\hline Acetophenone & 0.49 & 0.04 \\
Phenol & 0.33 & 0.61 \\
\hline
\end{tabular}

two standard compounds were selected, viz. acetophenone (a strong HBA) and phenol (a strong HBD). The solvatochromic parameters for their HBA and HBD ability were used as the physical quantities $\beta$ and $\alpha$, respectively [9]. These values are listed in Table I. From the retention indices of these two standards in a specific surfactant system and in an SDS system, $\Delta I_{S F}$ values can be calculated according Eq. (1) and the system constants $x$ and $y$ can be calculated applying Eq. (2). For five anionic and two cationic surfactant systems these constants $x$ and $y$ were calculated which are listed in Table II. With these constants the applied micellar systems can be classified according to their HBD strength $(x)$ as follows:

$$
\mathrm{TDS} \approx \mathrm{SDS}>\text { SDSo }>\text { SDS/Brij } 35 \approx \mathrm{CTAB} \approx \mathrm{DTAB}
$$

and according to their HBA strength $(y)$ as

$$
\mathrm{CTAB} \approx \mathrm{DTAB}>\mathrm{SDS} / \mathrm{Brij} 35>\text { SDSo }>\mathrm{SDS} \approx \mathrm{TDS}
$$

Here the mixed micellar system of $50 \mathrm{mM}$ SDS $/ 10 \mathrm{mM}$ Brij 35 was used. These results are comparable to a classification based on LSER models.

In order to illustrate the differences in selectivity between $50 \mathrm{mM}$ SDS and a mixed $50 \mathrm{mM}$ SDS $/ 10 \mathrm{mM}$ Brij 35 micellar system, in Table III the contributions to $\Delta I_{S D S / B r i j} 35$ of the hydrogen bonding terms $\beta x$ and $\alpha y$ are listed for 13 sample compounds possessing different functionalities. The negative values for $\beta x$ clearly illustrate the generally observed decrease in retention (i.e. negative $\triangle I_{S D S / B r i j} 35$ ) for strong HBA compounds in a mixed SDS/Brij 35 micellar system.

The reported approach has several advantages from a practical point of view. First, $\Delta I_{S F}$ values are calculated directly from MEKC migration data. Therefore these values provide a good insight into the influence of a specific micellar phase on MEKC migration behaviour and the expected position of the peaks in the electro-
Table III. Values for the hydrogen bonding terms $\beta x$ and $\alpha y$ for 13 sample compounds possessing different functionalities in a mixed micellar system of $50 \mathrm{mM}$ SDS/10 mM Brij 35 .

\begin{tabular}{|llr|}
\hline Compound & $\beta x$ & $\alpha y$ \\
\hline Phenol & -59 & 82 \\
Benzaldehyde & -79 & 0 \\
Nitrobenzene & -54 & 0 \\
Benzylcyanide & -67 & 0 \\
Acetophenone & -88 & 5 \\
Chlorobenzene & -13 & 0 \\
Naphtalene & -27 & 0 \\
Anilsol & -58 & 10 \\
Bromobenzene & -11 & 0 \\
$p$-Cresol & -61 & 78 \\
1,2-Xylol & -22 & 0 \\
Benzylalcohol & -94 & 53 \\
$p$-Nitrotoluene & -56 & 0 \\
\hline
\end{tabular}

kinetic chromatogram. Second, only for the standards retention indices must be determined. Consequently a relative small amount of experiments is required for the classification of pseudo-stationary phases. Third, only for the limited number of standard compounds solvatochromic parameters are required.

These results demonstrate that retention indices can be used for the quantitative characterization and classification of pseudo-stationary phases in MEKC in a similar way as the Rohrschneider-McReynolds scale in GC. We assume that this kind of classification may be helpful in the fast selection of an optimum composition of the micellar system for a given separation problem.

\section{References}

[1] S. Terabe, K. Otsuka, K. Ichikawa, A. Tsuchiya, T. Ando, Anal. Chem. 56, 111 (1984).

[2] S. Terabe, K. Otsuka, T. Ando, Anal. Chem. 57, 834 (1985).

[3] W. O. McReynolds, J. Chromatogr. Sci. 8, 685 (1970).

[4] L. R. Snyder, J. Chromatogr. Sci. 16, 223 (1978).

[5] S. Terabe, Y Okada, in "Proc. 15th Intern. Symp. on Capillary Chromatogr.", Hüthig Verlag, Heidelberg, 1993, p. 1420.

[6] S. Yang, M. Khaledi, Anal. Chem. 67, 499 (1995).

[7] S. Yang, M. Khaledi, J. Chromatogr. A, 692, 301 (1995).

[8] P. G. Muijselaar, H. A. Claessens, C. A. Cramers, Anal. Chem. 66, 635 (1994).

[9] M. J. Kamlet, R. M. Doherty, M. H. Abraham, Y. Marcus, R. W. Taft, J. Phys. Chem. 92, 5244 (1988).

Received: Nov 13, 1996 Accepted: Nov 18, 1996 